\title{
PREORDERS COMPATIBLE WITH PROBABILITY MEASURES DEFINED ON A BOOLEAN ALGEBRA
}

\author{
M. G. SCHWARZE
}

(Communicated by Thomas J. Jech)

\begin{abstract}
We use nonstandard analysis techniques to find conditions for the existence of a strictly positive measure weakly compatible with a preorder defined in a Boolean algebra generated by denumerably many atoms.
\end{abstract}

\section{INTRODUCTION}

When assigning probabilities, events can be considered as elements of a $\sigma$ complete Boolean algebra $B$ subject to the usual operation of union, intersection, complementation and $\sigma$-union.

Sometimes it is not possible to directly assign probabilities to events; however, it may be possible to compare events qualitatively with respect to a probability. Thus, we may be able to introduce a preorder $\lesssim$ on $B$ where $a \lesssim b$ can be intuitively interpreted as " $b$ is at least as probable as $a$ ".

It is natural then to ask what conditions on $B$ and $\lesssim$ are necessary and sufficient for the existence of a probability measure $\mu$ on $B$ such that for $p, q \in B, p \lesssim q$ iff $\mu(p) \leq \mu(q)$. If this is the case, we say that $\mu$ is compatible with $\lesssim$.

Villegas [4] found necessary conditions for the existence of a unique measure when $B$ is atomless, namely

(1) $\lesssim$ is a preorder on $B$, i.e. $p \lesssim q$ and $q \leqq r$ imply $p \lesssim r$, and $p \lesssim q$ or $q \lesssim p$ for $p, q, r \in B$;

(2) $\neg(1 \lesssim 0)$ and $0 \lesssim p$ for every $p \in B$;

(3) If $p, q, r \in B$ with $p \cap q \approx r \cap q \approx 0$ then $p \lesssim r$ iff $p \cup q \lesssim r \cup q$ ( $p \approx q$ is defined as $p \lesssim q$ and $q \lesssim p$ );

(4) If $p_{i}, q \in B$, and $p_{i} \subseteq p_{i+1} \lesssim q$ for every $i \in \mathbb{N}$, then $\bigcup p_{i} \leqslant q$.

He also proved that in any $\sigma$-complete Boolean algebra where (1)-(4) are satisfied there are at most denumerably many atoms and they can be ordered

Received by the editors October 19,1987. The contents of this paper were presented at the VII Latin American Logic Symposium held in Campinas, Brazil, in 1985. This research was funded by DIUC, grant $24 / 85$.

1980 Mathematics Subject Classification (1985 Revision). Primary 60A99; Secondary 03H10, $40 \mathrm{~J} 05$. 
in a decreasing sequence $a_{0} \gtrsim a_{1} \gtrsim a_{2} \gtrsim \cdots$, where $\gtrsim$ is defined by $p \gtrsim q$ iff $q \lesssim p$.

If $I$ is the ideal of all events $p$ with $p \approx 0$, it is easy to show, using the quotient algebra $B / I$, that it is enough to solve the problem for strictly positive compatible $\sigma$-measures, where a measure is strictly positive if $\mu(a)=0$ implies $a=0$.

In [3] Chuaqui and Schwarze claimed that conditions (1)-(4) were sufficient for the existence of a $\sigma$-measure compatible with a preorder on $B$ without the assumption that $B$ is atomless. This however is false.

In [2] Kraft, Pratt and Seidenberg gave an example of a Boolean algebra generated by 5 atoms where (1)-(3) and trivially (4) are satisfied but the algebra has no compatible measure. It is necessary to add one further condition:

(5) Every finite subalgebra $B^{\prime}$ of $B$ has a compatible measure.

With the methods used in [3] it is possible to show that conditions (1)-(5) are necessary and sufficient for the existence of a unique $\sigma$-measure compatible with a preorder defined on a $\sigma$-complete Boolean algebra in which $\bigcup a_{i} \lesssim \sim\left(\bigcup a_{i}\right)$.

The counterexample given in [2] also refutes a conjecture of de Finetti. He conjectured that for any finite preorder satisfying (1), (2), and (3) there exists a measure compatible with it. He also suggested that, if the conjecture is false, this is due to an inappropriate definition of the algebra $B$.

According to [2] it is possible to replace condition (5) by the following algebraic condition:

$\left(5^{\prime}\right)$ Let $B^{\prime}$ be any finite subalgebra of $B$.

Let $b_{0}, b_{1}, \ldots, b_{n-1}$ be the atoms of $B^{\prime}$. Let $p_{0}, p_{1}, \ldots, p_{m-1}, q_{0}, q_{1}, \ldots$, $q_{m-1}$ be elements of $B^{\prime}$ such that every $b_{i}$ is contained in the same number of $p_{j}$ 's and $q_{j}$ 's and $p_{i} \leqslant q_{i}$ for $i=1,2, \ldots, m-1$. Then $q_{0} \leqslant p_{0}$.

In [1] Chuaqui and Malitz studied the case where $B$ is an atomistic Boolean algebra.

The purely atomistic case has mathematical interest in its own right since, as Villegas proved, if there exists a compatible measure, there are at most countably many atoms, so that $B$ can be viewed as the power set of $\omega$, the set of natural numbers.

Chuaqui and Malitz were able to show that conditions (1)-(5) imply the existence of a weakly compatible strictly positive measure $\mu$ (i.e., a probability measure such that $p \lesssim q$ implies $\mu(p) \leq \mu(q))$, thus allowing the possibility that $p \prec q$ but $\mu(p)=\mu(q)$.

They also showed that when $B$ has a nonempty atomless part, conditions (1)-(5) imply the existence of a compatible measure.

When comparing events, sometimes the order obtained (by subjective or objective methods) is not total, so that we just have a partial preorder, where $\lesssim$ defined in $B$ is a partial preorder if $\lesssim$ is reflexive in $B$ and transitive (i.e., $p \lesssim p, p \lesssim q$ and $q \lesssim r$ implies $p \lesssim r$ for $p, q, r \in B)$. 
The main purpose of this work is to find conditions for the existence of a strictly positive $\sigma$-measure weakly compatible with a partial preorder defined in the $\sigma$-complete Boolean algebra $B$ of all subsets of $\omega$, the set of natural numbers. This will be accomplished using nonstandard analysis.

\section{Preliminaries}

Throughout this work $B$ will be the $\sigma$-complete Boolean algebra of all subsets of $\omega$. Let $\left\{a_{i}: i \in \omega\right\}$ be the set of atoms of $B$. $\lesssim$ will be a partial preorder defined on $B$ which extends $\subseteq$.

If $p \cap q=\mathbf{0}, p+q$ is $p \cup q$. If for all $i, j \in I$ with $i \neq j, p_{i} \cap p_{j}=\mathbf{0}$, $\sum\left\{p_{i}: i \in I\right\}=\bigcup\left\{p_{i}: i \in I\right\}$. Note that these operations are defined only for disjoint elements.

$p \prec q$ is defined as $p \lesssim q$ but $\neg(q \lesssim p)$.

$p \approx q$ is defined as $p \lesssim q$ and $q \lesssim p$.

Let $s_{n}$ be defined as $s_{n}=\sum\left\{a_{k}: k>n\right\}$. Such elements are called tails.

By a probability measure on $B$ we understand a $\sigma$-additive strictly positive real valued measure $\mu$ on $B$, such that $0<\mu(p) \leq \mu\left(\sum\left\{a_{i}: i \geq 0\right\}\right)<\infty$, for all $p \in B$ with $0<p$.

$\mu$ is compatible with $\lesssim$ iff for all $p, q \in B, p \lesssim q$ iff $\mu(p) \leq \mu(q)$.

$\mu$ is weakly compatible with $\lesssim$ if for all $p, q \in B, p \lesssim q$ implies $\mu(p) \leq$ $\mu(q)$

\section{MAIN THEOREM}

Throughout the discussion we will assume that $\lesssim$ has the following properties:

(1) Every finite subalgebra $B^{\prime}$ of $B$ generated by atoms and tails has a strictly positive measure weakly compatible with $\lesssim$.

(II) For all $k \in \omega$ there exists $k^{\prime} \in \omega$ such that $s_{k^{\prime}} \lesssim a_{k}$.

We will prove that conditions (I) and (II) are necessary and sufficient for the existence of a $\sigma$-measure strictly positive weakly compatible with $\lesssim$.

Condition (I) is obviously necessary, condition (II) is necessary to insure convergence.

Definition 1. For $p \in B$ let $p_{(n)}$ and $p^{(n)}$ be defined as follows:

$$
p_{(n)}=p \cap \sum\left\{a_{i}: i \leq n\right\}, \quad p^{(n)}=p \cup s_{n} .
$$

Notice that for all $n, p_{(n)} \subseteq p \subseteq p^{(n)}$.

Definition 2. $B_{1}=\left\{p^{(n)}: p \in B, n \in \omega\right\} \cup\left\{p_{(n)}: p \in B, n \in \omega\right\}$.

For all $p \in B_{1}$, let $c_{p}$ be a constant. Then (I) implies that

$$
\begin{aligned}
T= & \left\{c_{p} \leq c_{q}: p, q \in B_{1} \text { and } p \lesssim q\right\} \\
& \cup\left\{c_{p}=c_{q}+c_{r}: p, q, r \in B_{1} \text { and } p \approx q+r\right\} \cup \operatorname{Th} \mathbf{R}
\end{aligned}
$$

has a model $\mathfrak{M}$. 
We can assume $\mathfrak{M}$ is an $\aleph_{1}$-saturated nonstandard model of $T$.

For $p \in B$, we define $\mathbf{p} \in|\mathfrak{M}|$ in the following way:

(a) If there is a $q \in B_{1}$ such that $p \approx q$, let $\mathbf{p}=c_{q}^{\mathfrak{M}}$.

(b) If not, as $\mathfrak{M}$ is $\aleph_{1}$-saturated there exists $\left\{p_{n}\right\}_{n \in \mathbb{N}^{*}}$ and $\left\{p^{n}\right\}_{n \in \mathbb{N}^{N}}$ internal sequences such that $p_{n}=\mathbf{p}_{(\mathbf{n})}$ and $p^{n}=\mathbf{p}^{(\mathbf{n})}$ for $n \in \mathbb{N}$. Let $M=\left\{n \in{ }^{*} \mathbb{N}\right.$ : For $0 \leq k \leq n,\left\{p_{k}\right\}$ is increasing, $\left\{p^{k}\right\}$ is decreasing, and for all $r \leq n$ and $\left.k \leq n\left(p_{r}<p^{k}\right)\right\}$.

Then $M$ is internal and $\mathbb{N} \subseteq M$, so there must exist $\lambda \in M$, such that $\lambda \in{ }^{*} \mathbb{N}-\mathbb{N}$. We choose such a $\lambda$ and define $\mathbf{p}=p_{\lambda}$. It is clear that for all $p \in B, n \in \mathbb{N}, \mathbf{p}_{(\mathbf{n})} \leq \mathbf{p} \leq \mathbf{p}^{(\mathbf{n})}$.

Definition 3. Let $<_{1}$ and $\approx_{1}$ be defined on $B$, as follows: $p<_{1} q$ iff there exists $a_{k}$ such that $\mathbf{p}+\mathbf{a}_{\mathbf{k}}<\mathbf{q} ; p \approx_{1} q$ iff for all $a_{k},|\mathbf{p}-\mathbf{q}| \leq \mathbf{a}_{\mathbf{k}} \cdot p \lesssim_{1} q$ is defined as $p<_{1} q$ or $p \approx_{1} q$. It is clear that $p \lesssim_{1} q$ iff for all $k, \mathbf{p}<\mathbf{q}+\mathbf{a}_{\mathbf{k}}$.

Lemma 1. $\lesssim_{1}$ is a simple preorder.

Proof. We will prove first that $\lesssim_{1}$ is simple. If $\neg\left(p \approx_{1} q\right)$, then there exists $a_{k}$ such that $|\mathbf{p}-\mathbf{q}|>a_{k}$. Then $\mathbf{p}-\mathbf{q}>\mathbf{a}_{\mathbf{k}}$ and $q<_{1} p$, or $\mathbf{p}-\mathbf{q}<-\mathbf{a}_{\mathbf{k}}$ and $p<1 q$.

To show that $\lesssim_{1}$ is transitive, let $p, q, r \in B$ be such that $p \lesssim_{1} q$ and $q \lesssim_{1} r$. Given $a_{k}$, there exists $s_{k^{\prime}}$ such that $s_{k^{\prime}} \lesssim a_{k}$ and since $p \lesssim_{1} q$ and $q \lesssim_{1} r, \mathbf{p}<\mathbf{q}+\mathbf{a}_{\mathbf{k}^{\prime}+1}$ and $\mathbf{q}<\mathbf{r}+\mathbf{a}_{\mathbf{k}^{\prime}+2}$. Then $\mathbf{p}<\mathbf{r}+\mathbf{a}_{\mathbf{k}^{\prime}+1}+\mathbf{a}_{\mathbf{k}^{\prime}+2}<\mathbf{r}+\mathbf{s}_{\mathbf{k}^{\prime}}<$ $\mathbf{r}+\mathbf{a}_{\mathbf{k}}$, and therefore $p \lesssim_{1} r$.

Lemma 2. If $p \lesssim_{1} q$, then there exists $n \in \mathbb{N}, m \in \mathbf{N}$ such that $\mathbf{p}^{(\mathbf{n})}<\mathbf{q}_{(\mathbf{m})}$. Proof. There exists $a_{k}$ such that $\mathbf{p}+\mathbf{a}_{\mathbf{k}}<\mathbf{q}$. Let $m$ be such that $s_{m} \lesssim a_{k}$. Then $\mathbf{s}_{\mathbf{m}} \leq \mathbf{a}_{\mathbf{k}}$ and $\mathbf{p}+\mathbf{s}_{\mathbf{m}}<\mathbf{q}$. We can choose $s_{n}$ such that $s_{n} \lesssim a_{m+1}$, so

$$
\mathbf{p}+\mathbf{s}_{\mathrm{n}}+\mathbf{s}_{\mathbf{m + 1}}<\mathbf{p}+\mathbf{a}_{\mathbf{m}+1}+\mathbf{s}_{\mathbf{m + 1}}=\mathbf{p}+\mathbf{s}_{\mathbf{m}}<\mathbf{p}+\mathbf{a}_{\mathbf{k}}<\mathbf{q} \text {. }
$$

But $\mathbf{q} \leq \mathbf{q}^{(\mathbf{m}+1)}=\mathbf{q}_{(\mathbf{m}+1)}+\mathbf{s}_{\mathbf{m}+1}$ therefore $\mathbf{p}+\mathbf{s}_{\mathbf{n}}+\mathbf{s}_{\mathbf{m}+1}<\mathbf{q}_{(\mathbf{m}+1)}+\mathbf{s}_{\mathbf{m}+1}$ and thus $\mathbf{p}^{(\mathbf{n})}<\mathbf{q}_{(\mathbf{m}+\mathbf{1})}$.

Lemma 3. For all $p, q \in B_{1}$ we have that $p \lesssim q$ implies $p \lesssim_{1} q$.

Proof. If not, there exist $p, q \in B$ such that $p \lesssim q$ but $\neg\left(p \lesssim_{1} q\right)$. Then, Lemma 1 implies that $q \lesssim_{1} p$. Now Lemma 2 implies that there exists $n, m \in$ $\mathbf{N}$ such that $\mathbf{q}^{(\mathbf{n})}<\mathbf{p}_{(\mathbf{m})}$. But $p_{(m)} \lesssim p \lesssim q \lesssim q^{(n)}$ so $p_{(m)} \lesssim q^{(n)}$, also $p_{(m)}$, $q^{(n)} \in B_{1}$, therefore $\mathbf{p}_{\mathbf{m}}=\left(\mathbf{p}_{\mathbf{m}}\right)^{\mathfrak{M}} \leq\left(\mathbf{q}^{\mathbf{n}}\right)^{\mathfrak{M}}=\mathbf{q}^{\mathbf{n}}:$ a contradiction.

Lemma 4. For all $q, p_{i} \in B, i=1,2, \ldots$ with $p_{i} \subseteq p_{i+1} \lesssim_{1} q$. We have $\bigcup p_{i} \lesssim_{1} q$.

Proof. If not, there exist $q, p_{i} \in B$ with $p_{i} \subseteq p_{i+1} \lesssim_{1} q$ but $q \lesssim_{1} \cup p_{i}$. But then, there exist $k, r$ such that $\mathbf{q}^{(\mathbf{r})}<\left(\bigcup p_{i}\right)_{(\mathbf{k})}$. But $\left(\bigcup p_{i}\right)_{(k)}=\left(p_{n}\right)_{(k)}$ for some $n$, therefore $\mathbf{q} \leq \mathbf{q}^{(\mathbf{r})}<\left(\mathbf{p}_{\mathbf{n}}\right)_{(\mathbf{k})}$. 
If there exists $a_{t}$, with $a_{t} \subseteq \bigcup p_{i} \sim\left(p_{n}\right)_{(k)}$, then there exists $m$ such that

$$
\mathbf{q}+\mathbf{a}_{\mathbf{t}}<\left(\mathbf{p}_{\mathbf{n}}\right)_{(\mathbf{k})}+\mathbf{a}_{\mathbf{t}} \leq\left(\mathbf{p}_{\mathbf{n}}\right)_{(\mathbf{k})}+\mathbf{a}_{\mathbf{t}} \leq\left(\mathbf{p}_{\mathbf{m}}\right)_{(\mathbf{t})} \leq \mathbf{p}_{\mathbf{m}},
$$

which contradicts the fact that $p_{m} \lesssim_{1} q$.

If there is no such $a_{t}$, then $\bigcup p_{i}=p_{n}$. But $p_{n} \lesssim_{1} q$, therefore $\bigcup p_{i} \lesssim_{1} q$.

Lemma 5. Every finite subalgebra $B^{\prime}$ of $B$ has a strictly positive $\sigma$-measure $\mu$ compatible with $\aleph_{1}$.

Proof. Let $\left\{b_{0}, b_{1}, \ldots, b_{n-1}\right\}$ be the set of atoms of $B^{\prime}$. By [2] it is enough to show that for all elements $p_{0}, p_{1}, \ldots, p_{m-1}, q_{0}, \ldots, q_{m-1}$ such that each $b_{j}, j<n$, appears in the same number of $p_{i}$ 's and $q_{i}$ 's with $p_{i} \lesssim_{1} q_{i}$ for $i=1,2, \ldots, m-1$. Then $q_{0} \lesssim_{1} p_{0}$. Let

$$
\begin{aligned}
p_{i}=\alpha_{i 0} b_{0}+\alpha_{i 1} b_{1}+\cdots+\alpha_{i n-1} b_{n-1} ; \quad q_{i}=\beta_{i 0} b_{0}+\beta_{i 1} b_{1}+\cdots+\beta_{i n-1} b_{n-1}, \\
\text { for } i<m \text { and } \alpha_{i j}, \beta_{i j} \in\{0,1\} .
\end{aligned}
$$

By hypothesis we know $\sum_{\theta \leq j \leq m-1} \alpha_{i j}=\sum_{\theta \leq j \leq m-1} \beta_{i j}$, for $i<n$. Given $a_{k}$ there exists $s_{k^{\prime}}$ such that $s_{k^{\prime}} \lesssim a_{k}$.

$p_{i} \lesssim_{1} q_{i}$, therefore $\mathbf{p}_{\mathbf{i}}<\mathbf{q}_{\mathbf{i}}+\mathbf{a}_{\mathbf{k}^{\prime}+\mathbf{i}}, i<m$. We have $\sum_{1 \leq i \leq m-1}\left(\mathbf{p}_{\mathbf{i}}-\mathbf{q}_{\mathbf{i}}\right)<$ $\sum_{1 \leq i \leq m-1} \mathbf{a}_{\mathbf{k}^{\prime}+\mathbf{i}}<\mathbf{s}_{\mathbf{k}^{\prime}}<\mathbf{a}_{\mathbf{k}}$. But

$$
\begin{aligned}
\sum_{1 \leq i \leq m-1}\left(\mathbf{p}_{\mathbf{i}}-\mathbf{q}_{\mathbf{i}}\right)= & \sum_{1 \leq j \leq m-1}\left(\alpha_{j 0}-\beta_{j 0}\right) \mathbf{b}_{\mathbf{0}}+\sum_{1 \leq j \leq m-1}\left(\alpha_{j 1}-\beta_{j 1}\right) \mathbf{b}_{\mathbf{1}} \\
& +\cdots+\sum_{1 \leq j \leq m-1}\left(\alpha_{j n-1}-\beta_{j n-1}\right) \mathbf{b}_{\mathbf{n}-\mathbf{1}}
\end{aligned}
$$

and

$$
\sum_{1 \leq j \leq m-1}\left(\alpha_{j i}-\beta_{j i}\right)=\sum_{\theta \leq j \leq m-1} \alpha_{j i}-\alpha_{0 i}-\sum_{\theta \leq j \leq m-1} \beta_{j i}+\beta_{0 i}=\beta_{0 i}-\alpha_{0 i} .
$$

Therefore

$$
\left(\beta_{00}-\alpha_{00}\right) \mathbf{b}_{0}+\left(\beta_{01}-\alpha_{01}\right) \mathbf{b}_{1}+\cdots+\left(\beta_{0 n-1}-\alpha_{0 n-1}\right) \mathbf{b}_{\mathbf{n}-\mathbf{1}}<\mathbf{a}_{\mathbf{k}},
$$

so $\mathbf{q}_{\mathbf{0}}-\mathbf{p}_{\mathbf{0}}<\mathbf{a}_{\mathbf{k}}$. But $a_{k}$ was arbitrary, so $q_{0} \lesssim_{1} p_{0}$.

Lemmas 4 and 5 together with [1, Theorem 5.3] imply the existence of a strictly positive $\sigma$-measure $\mu$ weakly compatible with $\lesssim_{1}$; this together with Lemma 3 implies the following theorem.

Theorem. Let $B$ be a $\sigma$-complete Boolean algebra generated by countably many atoms $\left\{a_{i}: i \in \omega\right\}$ and $\lesssim$ a partial preorder on $B$ which extends $\subseteq$, such that

(I) Every finite subalgebra $B^{\prime}$ of $B$ generated by atoms and tails has a strictly positive measure weakly compatible with $\lesssim$.

(II) For any atom $a_{k}$ there exists a tail $s_{k^{\prime}}$ such that $s_{k^{\prime}} \leqslant a_{k}$.

Then there exists a strictly positive $\sigma$-measure $\mu$ weakly compatible with $\lesssim$.

\section{CountereXAMPLes}

Example 1. Let $B$ be a $\sigma$-complete Boolean algebra generated by $\left\{a_{i}: i \in \omega\right\}$. For $p \in B$ we define $\mathbf{p}$ in the following way: 
If $p=\sum\left\{a_{i}: i \in J\right\}$ with $J \subseteq \omega$, then $\mathbf{p}=\lim _{i \rightarrow \infty} \sum_{0 \leq k \leq i}\left[\chi_{J}(k)\right] \cdot$ $(1 / 2)^{k+1}$, where $\chi_{J}$ is the characteristic function of $J$.

Now we define $\lesssim$ on $B$. Let $p$ and $q$ be any elements of $B$.

(i) If $a_{0} \subsetneq p \cup q$, then $p \lesssim q$ iff $\mathbf{p} \leq \mathbf{q}$.

(ii) If $p \subsetneq \sum\left\{a_{i}: i>0\right\}$ and $a_{0} \subseteq q$, then $p \prec q$.

(iii) $a_{0} \prec \sum\left\{a_{i}: i>0\right\}$.

(iv) If $a_{0} \subseteq p \cap q$, then $p \lesssim q$ iff $\left(p-a_{0}\right) \lesssim\left(q-a_{0}\right)$.

Then $\lesssim$ is a total preorder; furthermore for all $n, s_{n+2} \lesssim a_{n}$ and every subalgebra $B^{\prime}$ of $B$ has a measure compatible with $\lesssim$. Therefore $B$ has a $\sigma$-measure weakly compatible with $\lesssim$. However, for any measure weakly compatible with $\lesssim, \mu\left(a_{0}\right)=\mu\left(\sum\left\{a_{i}: i>0\right\}\right)$ holds, so $B$ admits no compatible measure.

This example shows that strengthening condition (5) does not yield a stronger result. Namely, even if every finite subalgebra $B^{\prime}$ of $B$ has a measure compatible with $\lesssim, B$ admits only weakly compatible $\sigma$-measures.

The following two examples will show that condition (II) is necessary when the $\sigma$-algebra is infinite. In Example 2 the $\sigma$-algebra admits a finitely additive measure $\mu$ compatible with $\lesssim$, but no $\sigma$-additive measure weakly compatible with it. In Example 3 the $\sigma$-algebra does not admit even a finitely additive measure compatible with $\lesssim$.

Example 2. Let $B$ be a Boolean algebra generated by denumerably many atoms $\left\{a_{i}: i \in \omega\right\}$, and Cof the filter over $\omega$ such that $a \subset \omega$ belongs to Cof if and only if $\omega \sim a$ is finite.

Let $U$ be an ultrafilter over $\omega$ such that Cof $\subset U$. Let $\mu: B \rightarrow[0,1]$ be defined as:

$$
\mu(p)= \begin{cases}\frac{1}{2}+\sum\left\{\left(\frac{1}{2}\right)^{i+2}: a_{i} \subseteq p\right\}, & \text { if }\left\{i: a_{i} \subseteq p\right\} \in U, \\ \sum\left\{\left(\frac{1}{2}\right)^{i+2}: a_{i} \subseteq p\right\}, & \text { otherwise. }\end{cases}
$$

To show $\mu$ is finitely additive, let $p \cap q=0$. If $\left\{i: a_{i} \subseteq p\right\} \in U$, then $\left\{i: a_{i} \subseteq q\right\} \notin U$ and $\left\{i: a_{i} \subseteq p \cup q\right\} \in U$. So:

$$
\begin{aligned}
\mu(p)+\mu(q) & =\frac{1}{2}+\sum\left\{\left(\frac{1}{2}\right)^{i+2}: a_{i} \subseteq p\right\}+\sum\left\{\left(\frac{1}{2}\right)^{i+2}: a_{i} \subseteq q\right\} \\
& =\frac{1}{2}+\sum\left\{\left(\frac{1}{2}\right)^{i+2}: a_{i} \subseteq p \cup q\right\}=\mu(p \cup q) .
\end{aligned}
$$

Now if $\left\{i: a_{i} \subseteq p\right\} \notin U$ and $\left\{i: a_{i} \subseteq q\right\} \notin U$, then $\left\{i: a_{i} \subseteq p \cup q\right\}=$ $\left\{i: a_{i} \subseteq p\right\}+\left\{i: a_{i} \subseteq q\right\} \notin U$. So

$$
\begin{aligned}
\mu(p)+\mu(q) & =\sum\left\{\left(\frac{1}{2}\right)^{i+2}: a_{i} \subseteq p\right\}+\sum\left\{\left(\frac{1}{2}\right)^{i+2}: a_{i} \subseteq q\right\} \\
& =\sum\left\{\left(\frac{1}{2}\right)^{i+2}: a_{i} \subseteq p \cup q\right\}=\mu(p \cup q) .
\end{aligned}
$$


Then the order $\lesssim$ induced by $\mu$ has a compatible measure, but no $\sigma$-additive compatible measure, since for all $n \in \omega, \sum\left\{a_{i}: 0 \leq 1 \leq n\right\}<\sum\left\{a_{i}: 1>n\right\}$.

Example 3. Let $B$ be generated by denumerably many atoms $\left\{a_{i}: i \in \omega\right\}$. For all $p \in B$ let $\mathbf{p}$ be a constant. Let

$$
\begin{aligned}
T= & \left\{\mathbf{a}_{\mathbf{n}}=\mathbf{a}_{\mathbf{m}}: n, m \in \omega\right\} \cup\{\mathbf{p}<\mathbf{q}: p \subset q\} \\
& \cup\{\mathbf{p}=\mathbf{q}: p=q\} \cup\{\mathbf{p}=\mathbf{q}+\mathbf{r}: p=q+r\} .
\end{aligned}
$$

Then every finite subset $T^{\prime}$ of $T \cup$ ThR , has a model, then $T \cup$ ThR has a model $\mathfrak{M}$. Let $\lesssim$ be the preorder on $B$ defined by: $p \lesssim q$ iff $\mathbf{p}^{\mathfrak{M}} \leq \mathbf{q}^{\mathfrak{M}}$. As $\mathfrak{M}$ is a model of $T h R$, condition (I) is satisfied, but there exists no strictly positive measure weakly compatible with $\lesssim$ since for all $n, m, a_{n} \sim a_{m}$.

\section{BIBLIOGRAPHY}

1. R. Chuaqui and J. Malitz, Preorderings compatible with probability measures, Trans. Amer. Math. Soc. 279 (1983), 811-824.

2. C. H. Kraft, J. W. Pratt and A. Seidenberg, Intuitive probability on finite sets, Ann. Math. Statist. 30 (1959), 408-419.

3. M. G. Schwarze and R. Chuaqui, $\sigma$-additive measurement structures, Mathematical Logic in Latin America (Arruda, Chauqui and da Costa, eds.), North-Holland, Amsterdam, 1980, pp. 351-364.

4. C. Villegas, On qualitative probability $\sigma$-algebras, Ann. Math. Statist. 35 (1964), 1787-1796. CHILE

Department of Mathematics, Pontificia Universidad Catolica de Chile, Santiago, 